\title{
Analysis of the Closures of Sub-grid Scale Variance of Reaction Progress Variable for Turbulent Bunsen Burner Flames at Different Pressure Levels
}

\author{
Felix Benjamin Keil ${ }^{1} \cdot$ Nilanjan Chakraborty $^{2} \cdot$ Markus Klein $^{1}$ (D)
}

Received: 29 January 2020 / Accepted: 5 May 2020 / Published online: 24 May 2020

(c) The Author(s) 2020

\begin{abstract}
The statistical behaviour and modelling of the sub-grid variance of reaction progress variable have been analysed based on a priori analysis of direct numerical simulation (DNS) data of turbulent premixed Bunsen burner flames at different pressure levels. An algebraic expression for sub-grid variance, which can be derived based on a presumed bi-modal subgrid distribution of reaction progress variable with impulses at unburned reactants and fully burned products, has been found to be inadequate for the purpose of prediction of sub-grid variance even for the flames in the wrinkled flamelets/corrugated flamelets regime. Moreover, an algebraic model, which is often used for modelling sub-grid variance of passive scalars, has been found to significantly overpredict the sub-grid variance of reaction progress variable for all the cases considered here. The modelling of the unclosed terms of the sub-grid variance transport equation has been analysed in detail. Suitable model expressions have been identified for the sub-grid flux of variance, reaction rate contribution and scalar dissipation rate based on a priori analysis of DNS data. It has been found that the alternation of pressure does not have any significant impact on the closures of sub-grid flux of variance but a model parameter for the Favre-filtered scalar dissipation rate needs to be modified to account for the variation of pressure.
\end{abstract}

Keywords Sub-grid scale scalar variance $\cdot$ Bunsen burner premixed flames Elevated pressure $\cdot$ Direct numerical simulations

Markus Klein

markus.klein@unibw.de

Nilanjan Chakraborty

nilanjan.chakraborty@ncl.ac.uk

1 Department of Aerospace Engineering, Bundeswehr University Munich, Werner-Heisenberg-Weg 39, 85577 Neubiberg, Germany

2 School of Engineering, University of Newcastle, Claremont Road, Newcastle NE1 7RU, UK 


\section{Introduction}

In gas turbines and internal combustion engines, premixed turbulent combustion often takes place under elevated thermodynamic pressures. However, most combustion models have been proposed and validated at atmospheric conditions because of relative ease of conducting experiments and simulations for atmospheric pressure. The flame thickness decreases and turbulent Reynolds number increases (for a given set of values of root-mean-square velocity fluctuation and integral length scale) with increasing pressure and thus it often becomes challenging to analyse high-pressure premixed flames. The advances in high performance computing have enabled detailed analyses of high pressure premixed turbulent combustion and its associated modelling using direct numerical simulations (DNS) data (Savard et al. 2017; Klein et al. 2018a, b; Chakraborty et al. 2019; Alquallaf et al. 2019). The present paper focuses on the assessment of the closure of sub-grid scale (SGS) reaction progress variable variance under elevated pressure conditions for turbulent Bunsen burner flames. The SGS reaction progress variable variance $\sigma_{v}^{2}$ is defined as (Pierce and Moin 1998; Jimenez et al. 2001; Langella and Swaminathan 2016; Langella et al. 2016; Ranjan et al. 2016)

$$
\sigma_{v}^{2}=\widetilde{c^{2}}-\tilde{c}^{2}
$$

where $c$ is the reaction progress variable, and $\tilde{Q}=\overline{\rho Q} / \bar{\rho}$ and $\bar{Q}$ denote Favre-filtered and filtered value of a general variable $Q$, respectively with $\rho$ being the gas density.

The closure of sub-grid scalar variance plays a crucial role in the modelling of micro-mixing in the context of Large Eddy Simulations (LES) (Pierce and Moin 1998; Jimenez et al. 2001). Moreover, $\sigma_{v}^{2}$ is a quantity of pivotal importance in turbulent premixed combustion modelling (Langella and Swaminathan 2016; Langella et al. 2016; Ranjan et al. 2016). The present analysis utilises an existing DNS database of turbulent Bunsen flames at different thermodynamic pressures representing the flamelet regime of combustion [flames with low Karlovitz number (i.e. $K a<1$ )], which has been extended for this study by simulating a Bunsen flame with 25 times the baseline pressure. A recent study (Nilsson et al. 2019) focussed on the closure of the SGS variance $\sigma_{v}^{2}$ and its transport for high Karlovitz number flames but such an analysis is yet to be carried out for the low Karlovitz number conditions, located at the boundary of the wrinkled and the corrugated flamelets regimes, for a range of different thermodynamic pressures. This deficit in the existing literature has been addressed in the present work with a particular focus on the modelling of pressure effects on the unclosed terms in the transport equation of $\sigma_{v}^{2}$ for a range of different filter widths.

\section{Mathematical Background and Numerical Implementation}

An algebraic expression is often used for the evaluation of SGS variance of passive scalars in the following manner (Pierce and Moin 1998; Langella and Swaminathan 2016; Ranjan et al. 2016):

$$
\sigma_{v}^{2}=C \Delta^{2}|\nabla \tilde{c}|^{2}
$$

where $C=0.5$ is the model constant (Ranjan et al. 2016) and $\Delta$ is the LES filter width. It is expected that $\sigma_{v}^{2}$ is affected by both chemical and turbulent processes (Chakraborty and Swaminathan 2011; Lai and Chakraborty 2016), which is not accounted for in Eq. 2. It is worth noting that $\sigma_{v}^{2}$ assumes the following expression: 


$$
\sigma_{v}^{2}=\tilde{c}(1-\tilde{c})+O\left(D a_{\Delta}^{-1}\right)
$$

subject to the assumption of a bimodal sub-grid probability density function (PDF) of $c$ with impulses at $c=0.0$ and $c=1.0$. Here $D a_{\Delta}=\Delta S_{\mathrm{L}} / u_{\Delta}^{\prime} \delta_{t h}$ is the sub-grid Damköhler number with $u_{\Delta}^{\prime}=\sqrt{\left(\widetilde{u_{i} u_{i}}-\tilde{u}_{i} \tilde{u}_{i}\right) / 3}=\sqrt{2 k_{s g s} / 3}$ and $k_{s g s}=\left(\widetilde{u_{i} u_{i}}-\tilde{u}_{i} \tilde{u}_{i}\right) / 2$ being the SGS velocity fluctuation and the SGS turbulent kinetic energy, respectively. The term $O\left(D a_{\Delta}^{-1}\right)$ can be ignored only for $D a_{\Delta} \gg 1.0$, and under that condition, the maximum possible value of the SGS variance (i.e. $\sigma_{v}^{2}=\tilde{c}(1-\tilde{c})$ ) is obtained. However, the sub-grid PDF of $c$ may not be bi-modal (Bray et al. 1985) and it might be necessary to solve a modelled transport equation of $\sigma_{v}^{2}$ in the cases where Eq. 2 and $\sigma_{v}^{2}=\tilde{c}(1-\tilde{c})$ do not provide satisfactory performance. The exact transport equation of $\sigma_{v}^{2}$ is given by:

$$
\begin{aligned}
\frac{\partial\left(\bar{\rho} \sigma_{v}^{2}\right)}{\partial t}+\frac{\partial\left(\bar{\rho} \tilde{u}_{j} \sigma_{v}^{2}\right)}{\partial x_{j}}= & \underbrace{-\frac{\partial}{\partial x_{j}}\left[\overline{\rho u_{j} c^{2}}-2\left[\overline{\rho u_{j} c}-\bar{\rho} \tilde{u}_{j} \tilde{c}\right] \tilde{c}-\bar{\rho} \tilde{u}_{j} \tilde{c}^{2}\right]}_{T_{1}} \\
& \underbrace{-2\left[\overline{\rho u_{j} c}-\bar{\rho} \tilde{u}_{j} \tilde{c}\right] \frac{\partial \tilde{c}}{\partial x_{j}}}_{T_{2}} \underbrace{+2[\overline{\dot{w} c}-\overline{\dot{w}} \tilde{c}]}_{T_{3}}+\underbrace{\frac{\partial}{\partial x_{j}}\left(\rho D_{c} \frac{\partial \sigma_{v}^{2}}{\partial x_{j}}\right)}_{T_{4}} \underbrace{-2 \bar{\rho} \tilde{\epsilon}_{c}}_{D_{v}}
\end{aligned}
$$

where $u_{j}, \dot{w}$ and $D_{c}$ are the jth component of velocity, reaction rate of progress variable and reaction progress variable diffusivity, respectively. In Eq. 4, $\widetilde{\tilde{\epsilon}_{c}}=\overline{\rho D_{c} \nabla c \cdot \nabla c} / \bar{\rho}-\widetilde{D_{c}} \nabla \tilde{c} \cdot \nabla \tilde{c}$ is the sub-grid scalar dissipation rate (SDR), whereas $\widetilde{N_{c}}=\overline{\rho D_{c} \nabla c \cdot \nabla c} / \bar{\rho}$ will henceforth be referred to as the Favre-filtered SDR in this paper. The term $T_{1}$ accounts for turbulent transport of SGS variance, whereas the term $T_{2}$ denotes generation/destruction of SGS variance due to $\nabla \tilde{c}$. The term $T_{3}$ accounts for generation/ destruction of SGS variance due to chemical reaction rate, whereas $T_{4}$ depicts molecular diffusion of $\sigma_{v}^{2}$. The term $D_{v}=-2 \bar{\rho} \widetilde{\epsilon_{c}}$ originates due to molecular dissipation of SGS variance under the action of SDR. In LES, the SGS flux of reaction progress variable $\left[\overline{\rho u_{j} c}-\bar{\rho} \tilde{u}_{j} \tilde{c}\right]$ is modelled in order to solve the transport equation of $\tilde{c}$, and thus the term $T_{2}$ can be considered to be closed. The modelling of $\left[\overline{\rho u_{j} c}-\bar{\rho} \tilde{u}_{j} \tilde{c}\right]$ has been discussed elsewhere (Gao et al. 2015a, b; Klein et al. 2016; 2018c) and thus is not repeated here. The molecular diffusion term $T_{4}$ is also a closed term and thus the closure of the transport equation of $\sigma_{v}^{2}$ depends on the modelling of $T_{1}, T_{3}$ and $D_{v}$. In the present analysis, the terms of Eq. 4 have been extracted to analyse their statistical behaviours by explicitly filtering the DNS data and $T_{1}, T_{3}$ and $D_{v}$ extracted from DNS data have been utilised for assessment of their respective models.

For the purpose of this analysis, 6 different turbulent premixed Bunsen burner flames have been considered and these flames are taken from a database (which has been extended for this study) consisting of 16 different cases (Klein et al. 2018b). The chemical mechanism is simplified here using a single step irreversible reaction for the sake of computational economy so that a detailed parametric analysis involving different thermodynamic pressures can be conducted. The Reynolds number $R e=U_{B} d_{n} / v_{u}$ (based on the bulk inlet velocity $U_{B}$, nozzle diameter $d_{n}$, and the unburned-gas kinematic viscosity $v_{u}$ ); turbulent Reynolds number $R e_{t}=u^{\prime} l / \nu_{u}$; integral length scale to thermal flame thickness ratio $l / \delta_{t h}$; Damköhler number $D a=l S_{L} / \delta_{t h} u^{\prime}$; and Karlovitz number $K a=\left(u^{\prime} / S_{L}\right)^{3 / 2}\left(l / \delta_{t h}\right)^{-1 / 2}$ are listed in Table 1. Here $\delta_{t h}=\left(T_{a d}-T_{0}\right) / \max |\nabla T|_{L}$ is the thermal flame thickness with 
$T_{a d}$ and $T_{0}$ being the adiabatic flame temperature and the unburned reactant temperature, respectively, and the subscript ' $L$ ' refers to the unstrained laminar flame quantities. The normalised mean inlet velocity is set to $U_{B} / S_{L}=6.0$ and the normalised turbulent rootmean-square (rms) velocity fluctuation equals $u^{\prime} / S_{L}=1.0$, except for case $\mathrm{D}$ where $u^{\prime} / S_{L}$ at the inlet is 3.10. It is worth noting that there was a special reason for considering moderate turbulence intensities: due to an increasing ratio of the hydrodynamic to critical length scale, the flames become increasingly hydrodynamically unstable (e.g. Darrieus-Landau (DL) instability) with increasing pressure, which is an important point for modelling high pressure turbulent premixed flames. As the effects of DL instability are masked by turbulence effects for high turbulence intensities, a small value of normalised turbulent rootmean-square (rms) velocity fluctuation $\left(u^{\prime} / S_{L}\right)$ has been used in this work in order to be able to analyse the effects of DL instability at elevated pressure levels. The integral length scale to Bunsen burner nozzle diameter ratio is given by $l / d_{n}=1 / 5$ except for case $\mathrm{E}$ where $l / d_{n}$ is $3 / 5$. For case $\mathrm{F}$ this corresponds to a ratio of $l / \delta_{t h}=25$ and in this sense the present database has much more realistic scale separation in terms of integral scale to thermal flame thickness $\left(l / \delta_{t h}\right)$ than most existing DNS databases where $l$ is of the same order as $\delta_{t h}$. The heat release parameter $\tau=\left(T_{a d}-T_{0}\right) / T_{0}$ and the Zel'dovich number $\beta=T_{a c}\left(T_{a d}-T_{0}\right) / T_{a d}^{2}$ are considered to be 4.5 and 6.0 respectively, and $T_{a c}$ is the activation temperature. Standard values of Prandtl number $(\operatorname{Pr}=0.7)$ and ratio of specific heats $\left(\gamma_{g}=1.4\right)$ have been used. All non-dimensional numbers in Table 1 are to be understood as the inlet values.

It can be noted from Table 1 that the turbulent Reynolds number $R e_{t}$ increases from case A to case $\mathrm{C}$ to F. Furthermore, it can be seen from Table 1 that cases $\mathrm{C}, \mathrm{D}$ and $\mathrm{E}$ have same values of $R e_{t}$ but cases $\mathrm{D}$ and $\mathrm{E}$ have one tenth of the pressure of that of case $\mathrm{C}$. The value of $u^{\prime} / S_{L}$ is higher in case $\mathrm{D}$ than in case $\mathrm{C}$ and $\mathrm{E}$, whereas $u^{\prime} / S_{L}$ and $l / \delta_{t h}$ values are exactly the same for cases $\mathrm{C}$ and $\mathrm{E}$ and thus they fall on the same point on the regime diagram (but behave very differently in terms of flame morphology). Although turbulent Reynolds number remains moderate for the cases considered here, it has been shown in the past that the model parameters for SDR reach an asymptotic value for $R e_{t} \approx 50.0$ (Chakraborty and Swaminathan 2013). Moreover, SDR models proposed using a priori analysis of moderate values of DNS data (Dunstan et al. 2013; Gao et al. 2014a) have been found to be perform well for flow configurations with much larger turbulent Reynolds number $R e_{t}$ based on a posteriori analysis using LES (Ma et al. 2014a).

The simulations of high pressure Bunsen flames have been conducted by adjusting the viscosity and the Arrhenius parameters in such a way that $S_{L} \sim P^{-0.5}$, and $v_{u} \sim P^{-1}$ are satisfied, as that of methane-air flames (Turns 2011). Thus, the Zeldovich flame thickness $\delta_{Z}$ scales as $\delta_{Z} \sim P^{-0.5}$ (where $\delta_{Z}=\alpha_{T 0} / S_{L}$ with $\alpha_{T 0}$ being the thermal diffusivity in the unburned gas) and the numerical resolution must be adjusted accordingly. The

Table 1 The turbulence inlet flow parameters for the considered cases

\begin{tabular}{lcrlrlc}
\hline Case & $P / P_{0}$ & $R e_{D}$ & $R e_{t}$ & $l / \delta_{t h}$ & $K a$ & $D a$ \\
\hline A & 1 & 399 & 13.30 & 5.20 & 0.45 & 5.00 \\
B & 5 & 892 & 29.26 & 11.40 & 0.30 & 11.40 \\
C & 10 & 1262 & 41.22 & 16.13 & 0.25 & 16.13 \\
D & 1 & 399 & 41.22 & 5.20 & 2.40 & 1.670 \\
E & 1 & 399 & 41.22 & 16.13 & 0.25 & 16.13 \\
F & 25 & 1262 & 64.89 & 26.0 & 0.20 & 26.00 \\
\hline
\end{tabular}


dimensions of the simulation domain are kept unchanged for all cases considered here and the nozzle diameter $d_{n}$ corresponds roughly to half the domain size. Simulation domains of size $50 \delta_{t h} \times 50 \delta_{t h} \times 50 \delta_{t h}$ for $P=P_{0}, 112 \delta_{t h} \times 112 \delta_{t h} \times 112 \delta_{t h}$ for $P=5 P_{0}$, $159 \delta_{t h} \times 159 \delta_{t h} \times 159 \delta_{t h}$ for $P=10 P_{0}$ and $250 \delta_{t h} \times 250 \delta_{t h} \times 250 \delta_{t h}$ for $P=25 P_{0}$ have been considered here, where $P_{0}$ is taken to be 1.0 bar (i.e. $P_{0}=1.0$ bar). Uniform Cartesian meshes of $250 \times 250 \times 250,560 \times 560 \times 560,795 \times 795 \times 795$ and $1272 \times 1272 \times 1272$ are used for cases A, D, E, case B, case C and case D, respectively, which ensure for all cases the resolution of the flame thickness and the smallest scales of turbulence (Klein et al. 2018a, b; Chakraborty et al. 2019; Alquallaf et al. 2019).

The simulations have been carried out using the DNS code SENGA (Jenkins and Cant 1999 ) in which the governing equations are solved using high order finite difference and Runge-Kutta time-advancement. Inflow data has been generated using a parallelised and modified version of the digital filter-based inflow methodology proposed by Klein et al. (2003) where the Gaussian filter in the temporal space has been replaced by an autoregressive AR1 process in order to avoid excessive filter length in this direction caused by the small-time step in the compressible flow solver. Interested readers are referred to Klein et al. (2018a) for further information regarding the DNS database considered here and its numerical implementation. The reacting scalar and flow fields have been initialised using an unstrained premixed laminar flame solution, which is specified as a function of radius from the nozzle centre. All the domain faces except for the inlet are specified using the partially non-reflecting NSCBC formalism (Poinsot and Lele 1992). The simulation time, when statistics were first taken, is chosen to be larger than two flow-through which corresponds to nearly four eddy-turnover times.

In this analysis, the DNS data has been explicitly LES filtered using a Gaussian filter kernel $G(\boldsymbol{r})$ so that the LES filtered values of a general quantity $Q$ can be calculated as follows (Peters 2000; Nilsson et al. 2019; Chakraborty and Swaminathan 2011; Lai and Chakraborty 2016; Dunstan et al. 2013; Gao et al. 2014a; Ma et al. 2014a; Boger et al. 1998; Reddy and Abraham 2012; Chakraborty and Cant 2007, 2009):

$$
\overline{Q(\boldsymbol{x})}=\int Q(\boldsymbol{x}-\boldsymbol{r}) G(\boldsymbol{r}) d \boldsymbol{r}, \quad G(\boldsymbol{r})=\left(6 / \pi \Delta^{2}\right)^{3 / 2} \exp \left(-6 \boldsymbol{r} \cdot \boldsymbol{r} / \Delta^{2}\right)
$$

Results will be presented from $\Delta \approx 0.03 d_{n}$ (corresponding for case A to the smallest filter width to flame thickness ratio of $\Delta / \delta_{t h}=0.8$ ) where the flame is partially resolved, up to $\Delta \approx 0.15 d_{n}$ (corresponding for case $\mathrm{F}$ to the largest filter width to flame thickness ratio $\Delta / \delta_{t h}=19.0$ ) where the flame becomes fully unresolved and $\Delta$ becomes comparable to the integral length scale $l$.

\section{Results and Discussion}

The isosurfaces of $\tilde{c}=0.5$ for cases $\mathrm{A}, \mathrm{C}, \mathrm{E}$ and $\mathrm{F}$ for $\Delta / d_{n}=0.06$ and 0.15 are exemplarily shown in Fig. 1 along with instantaneous views of $c=0.5$ isosurfaces of the corresponding cases. It can be seen from Fig. 1 that the flame morphology changes significantly with increasing pressure. The extent of wrinkling in cases $\mathrm{C}$ and $\mathrm{F}$ is significantly greater than in cases A and E due to DL instability (Klein et al. 2018a, b; Alquallaf et al. 2019) and interested readers are referred to Klein et al. (2018a, b) and Alquallaf et al. (2019) for further discussion on this aspect. Figure 1 further shows that the extent of wrinkling of the isosurface decreases with increasing $\Delta$ because of the smearing of local information due 
Fig. 1 Isosurfaces of cases A, C, $\mathrm{E}$ and $\mathrm{F}$ for $\Delta / d_{n}=0.06$ and 0.15 along with instantaneous views of $c=0.5$ case A

$c=0.5$

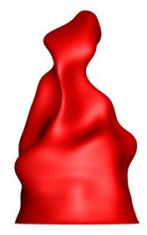

case $\mathrm{C}$

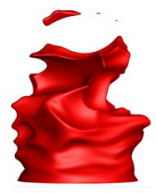

case E

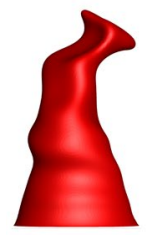

case $\mathrm{F}$
$\Delta / d_{n}=0.06$

$\Delta / d_{n}=0.15$
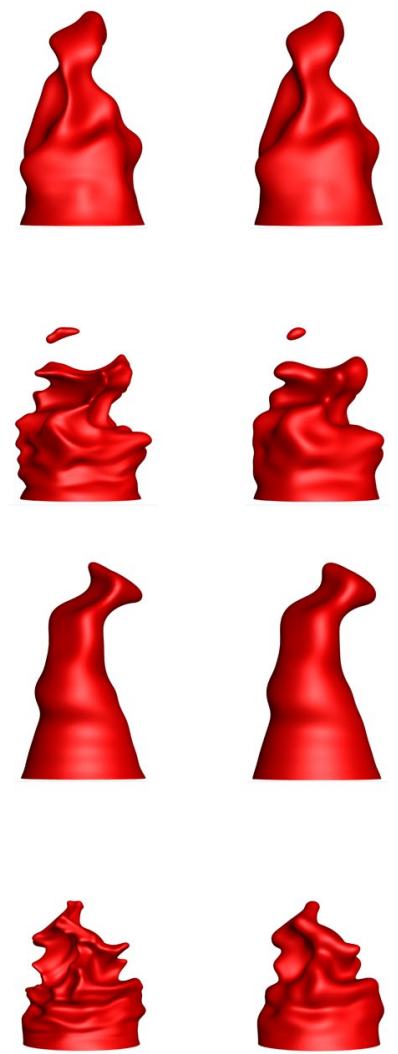

to the convolution operation associated with the filtering. For the sake of brevity, results will be shown for cases A, C and F only but results for cases B, D and E are qualitatively similar.

The variations of mean values of $\sigma_{v}^{2}$ conditional upon bins of $\tilde{c}$ for cases A, C, F for different values of $\Delta / d_{n}$ are shown in Fig. 2. It can be seen from Fig. 2 that $\sigma_{v}^{2}$ increases with increasing $\Delta$ before approaching an asymptotic value which is considerably smaller than the maximum possible value given by $\tilde{c}(1-\tilde{c})$. The variation of mean values of $D a_{\Delta}$ conditional upon bins of $\tilde{c}$ for cases $\mathrm{A}, \mathrm{C}, \mathrm{F}$ for different normalised filter widths $\Delta / d_{n}$ is shown in Fig. 3, which shows that $D a_{\Delta}$ increases with increasing $\Delta$ but $D a_{\Delta}$ does not assume large (i.e. $D a_{\Delta} \gg 1$ ) values in all cases even for the largest value of $\Delta$ considered here. This is consistent with the analysis in Keil et al. (2019). Thus, the $O\left(D a_{\Delta}^{-1}\right)$ contribution can only be ignored for $D a_{\Delta} \gg 1$ and under that condition the maximum possible value of the SGS variance $\left(\right.$ i.e. $\left.\sigma_{v}^{2}=\tilde{c}(1-\tilde{c})\right)$ is obtained. Differences between $\sigma_{v}^{2}$ and $\tilde{c}(1-\tilde{c})$ indicate the extent of the departure of the sub-grid PDF of $c$ from the bi-modal distribution with impulses at $c=0.0$ and 1.0, except for the largest filter width for case $\mathrm{F}$ which corresponds to $\Delta / \delta_{t h}=19$. This is consistent with previous findings (Dunstan et al. 2013; Gao et al. 2014a; Ma et al. 2014a; Chakraborty and Cant 2007, 2009).

In fact, Eq. 2 significantly overpredicts $\sigma_{v}^{2}$ and provides an unphysical prediction (i.e. $\sigma_{v}^{2}>\tilde{c}(1-\tilde{c})$ ) for all filter widths for the model parameter $C=0.5$. Moreover, the ratio 

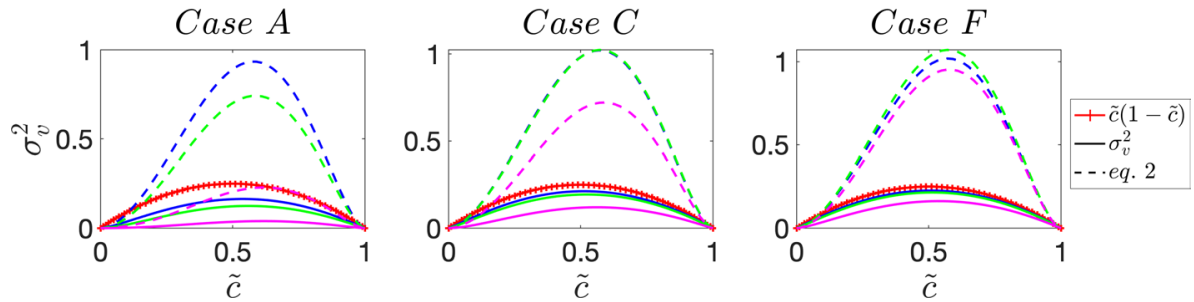

Fig. 2 Variations of mean values of $\sigma_{v}^{2}$ conditional upon bins of $\tilde{c}$ along with the predictions of Eq. 2 and $\tilde{c}(1-\tilde{c})$ for $\Delta / d_{n}=0.03$ (magenta), $\Delta / d_{n}=0.09$ (green) and $\Delta / d_{n}=0.15$ (blue) in cases A, C, F
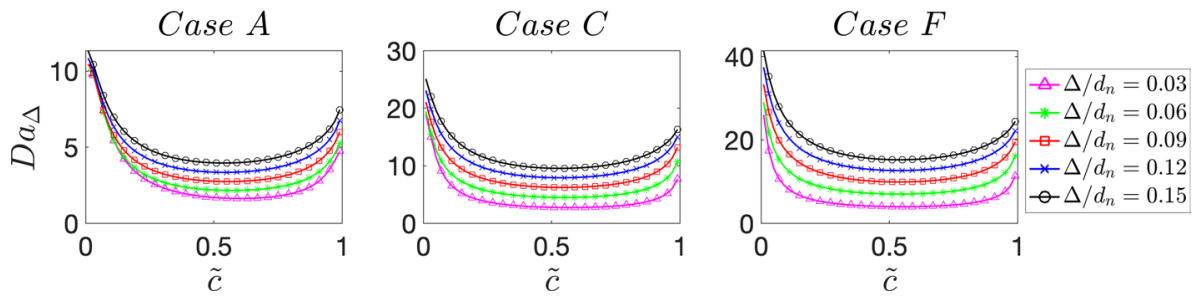

Fig. 3 Variations of mean values of $D a_{\Delta}$ conditional upon bins of $\tilde{c}$ for different $\Delta / d_{n}$ in cases A, C, F

$\sigma_{v}^{2} / \Delta^{2}|\nabla \tilde{c}|^{2}$ has been found to increase with increasing $\Delta$ and these values are different for each of the cases considered here (not shown). Thus, an alteration of $C$ from 0.5 to a different value will not be sufficient for algebraic closure of $\sigma_{v}^{2}$ for all cases because the optimum value of $C$ will not be a priori known.

The variations of $\left\{T_{1}, T_{2}, T_{3}, T_{4}\right.$ and $\left.D_{v}\right\} \times \delta_{t h} / \rho_{0} S_{L}$ (with $\rho_{0}$ being the unburned gas density) conditional upon bins of $\tilde{c}$ for $\Delta / d_{n}=0.03$ and 0.15 are shown in Fig. 4 for cases A, C, F. It can be seen from Fig. 4 that $D_{v}$ acts as a leading order sink term for all cases irrespective of the filter width. By contrast, the reaction rate contribution $T_{3}$ acts as a leading order source term apart from the negative contribution towards the burned gas side of the flame brush for all cases for all filter widths considered here. For small values of $\Delta, \widetilde{\epsilon_{c}}=\overline{\rho D_{c} \nabla c \cdot \nabla c} / \bar{\rho}-\tilde{D} \nabla \tilde{c} \cdot \nabla \tilde{c}$ tends to disappear as both $\overline{\rho D_{c} \nabla c \cdot \nabla c} / \bar{\rho}$ and $\tilde{D}_{c} \nabla \tilde{c} \cdot \nabla \tilde{c}$ approach $D_{c} \nabla c \cdot \nabla c$ (i.e. $\lim _{\Delta \rightarrow 0} \overline{\rho D_{c} \nabla c \cdot \nabla c} / \bar{\rho}=D_{c} \nabla c \cdot \nabla c$ and $\left.\lim _{\Delta \rightarrow 0} \tilde{D}_{\mathrm{c}} \nabla \tilde{c} \cdot \nabla \tilde{c}=D_{c} \nabla c \cdot \nabla c\right)$ and as a result of this the magnitude of the mean contribution of $D_{v}$ remains small for small filter widths but the relative magnitude of $D_{v}$ in comparison to $T_{3}$ increases with increasing $\Delta$ as the sub-grid SDR $\widetilde{\epsilon_{c}}$ increases with an increase in filter width.

The mean contribution of the resolved scalar gradient term $T_{2}$ remains negative for all cases for all filter widths which is indicative of counter-gradient transport (i.e. $\left[\overline{\rho u_{j} c}-\bar{\rho} \tilde{u}_{j} \tilde{c}\right] \partial \tilde{c} / \partial x_{j}>0$ ). The cases considered here are subjected to weak turbulence and thus the value of Bray number $N_{B}=\tau S_{L} / u^{\prime}$ remains greater than unity (i.e. $N_{B}>1.0$ ) for all cases. This suggests that the effects of flame normal acceleration dominate over those of turbulent velocity fluctuations leading to a dominant counter-gradient transport (Veynante et al. 1997). The magnitude of the mean contribution of $T_{2}$ remains smaller than that of $T_{3}$ for all cases considered here. The mean contribution of the molecular diffusion term $T_{4}$ remains positive on both unburned and burned gas sides of the flame brush with a negative dip in the middle of the flame brush in all cases irrespective of $\Delta$, whereas the qualitative behaviour of 

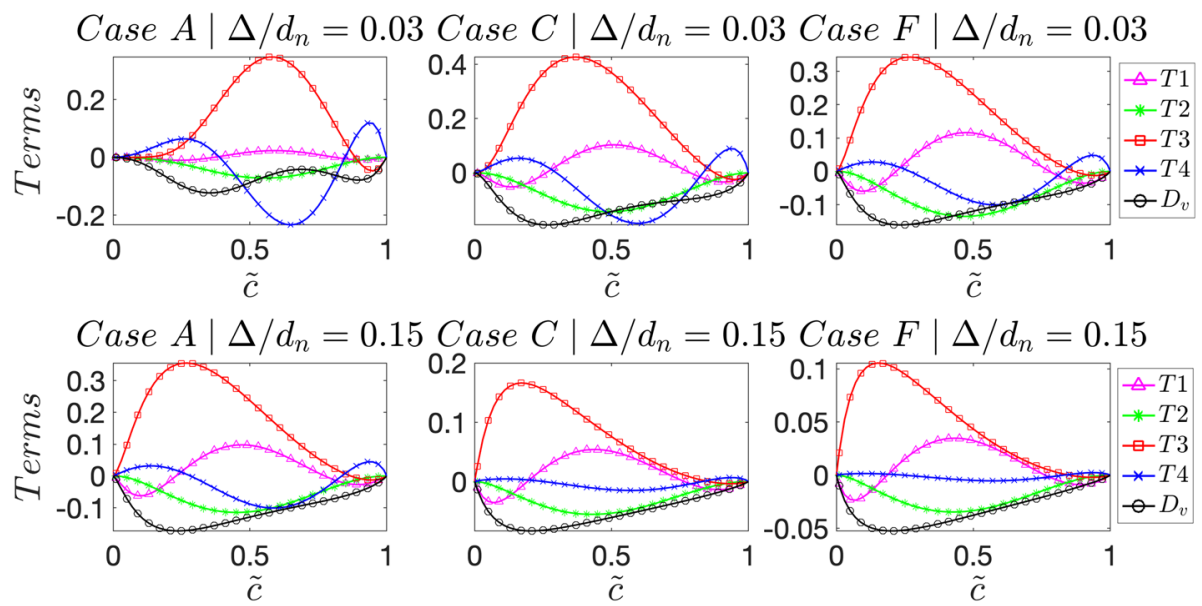

Fig. 4 Variations of mean values of $\left\{T_{1}, T_{2}, T_{3}, T_{4}\right.$ and $\left.D_{v}\right\} \times \delta_{t h} / \rho_{0} S_{L}$ conditional upon bins of $\tilde{c}$ for $\Delta / d_{n}=0.03$ and 0.15 in cases $\mathrm{A}, \mathrm{C}, \mathrm{F}$

the mean values of the turbulent transport term $T_{1}$ remains just the opposite to that of $T_{4}$. This behaviour originates due to counter-gradient behaviour of the SGS flux of scalar variance, which will be discussed later in this paper. The magnitudes of mean contributions of $T_{4}$ in comparison to the magnitudes of $D_{v}$ decrease with increasing $\Delta$ for all cases. It can further be seen from Fig. 4 that $T_{3}$ and $D_{v}$ remain of the similar order of magnitude for large $\Delta$ (e.g. $\Delta / d_{n}=0.15$ where $\Delta \gg \delta_{t h}$ ) but this equilibrium is not maintained for small filter sizes (e.g. $\Delta / d_{n}=0.03$ where $\left.\Delta \leq \delta_{t h}\right)$.

The observed mean behaviours of $T_{1}, T_{2}, T_{3}, T_{4}$ and $D_{v}$ have been found to be consistent with scaling estimates presented in Nilsson et al. (2019) and thus are not presented here. Moreover, the qualitative behaviours of the mean behaviours of $T_{1}, T_{2}, T_{3}, T_{4}$ and $D_{v}$ have been qualitatively similar to those previously reported for high Karlovitz number detailed chemistry DNS (Nilsson et al. 2019) and simple chemistry DNS (Keil et al. 2019) data for the flames representing the thin reaction zones regime combustion. The mean behaviors of $\left\{T_{1}, T_{2}, T_{3}, T_{4}\right.$ and $\left.D_{v}\right\} \times \delta_{t h} / \rho_{0} S_{L}$ conditional upon bins of $\tilde{c}$ for $\Delta / \delta_{t h}=0.8$ and 4.0 in cases A, C, F are shown in the "Appendix". It becomes obvious that the behavior of the terms in the transport equation of $\sigma_{v}^{2}$ is very similar for different pressures when the same filter width to thermal flame thickness ratio is chosen. However, for a real LES simulation that will mean that the computational time increases drastically because the mesh size has to follow the decreasing flame thickness with increasing pressure.

Equation 4 indicates that the closure of $T_{1}$ depends on the appropriate closure of the SGS flux of variance $F_{j}=\left[\overline{\rho u_{j} c^{2}}-2\left[\overline{\rho u_{j} c}-\bar{\rho} \tilde{u_{j}} \tilde{c}\right] \tilde{c}-\bar{\rho} \tilde{u_{j}} \widetilde{c^{2}}\right]$. According to the gradient hypothesis model (GHM) (Langella and Swaminathan 2016; Langella et al. 2016) one gets:

$$
F_{j}=\left[\overline{\rho u_{j} c^{2}}-2\left[\overline{\rho u_{j} c}-\bar{\rho} \tilde{u_{j}} \tilde{c}\right] \tilde{c}-\bar{\rho} \tilde{u_{j}} \tilde{c}^{2}\right]=-\bar{\rho} S c_{t}^{-1}\left(C_{s} \Delta\right)^{2} \sqrt{2 \tilde{S}_{i j} \tilde{S}_{i j}} \partial \sigma_{v}^{2} / \partial x_{j}
$$

where $C_{s}=0.18$ is the Smagorinsky constant, $\tilde{S}_{i j}=0.5\left(\partial \tilde{u}_{i} / \partial x_{j}+\partial \tilde{u}_{j} / \partial x_{i}\right)$ is the resolved strain rate and $S c_{t}$ is the turbulent $S c h m i d t$ number. The mean values of $F_{n} / \rho_{0} S_{L}$ (where $F_{n}$ is the projection of the Flux $F_{j}$ in resolved flame normal direction $\left.-\nabla \tilde{c} /|\nabla \tilde{c}|\right)$ conditional 
upon bins of $\tilde{c}$ are shown along with the prediction of $F_{j}^{G H M}$ (for $S c_{t}=1.0$ ) in Fig. 5 for cases A, C, F for $\Delta / d_{n}=0.03$ and 0.15 . Figure 5 shows that $F_{j}^{G H M}$ does not capture the qualitative behaviour of $F_{n}$ and it predicts the opposite sign to the value obtained from DNS, which is indicative of the counter-gradient behaviour of the SGS variance flux. These findings are expected for these flames because the effects of flame normal acceleration are likely to overcome the influences of turbulent fluctuation to yield counter-gradient transport for small values of $u^{\prime} / S_{L}$ (Gao et al. 2015a, b; Klein et al. 2016, 2018c; Veynante et al. 1997). This suggests that it will be desirable to have a model which is capable of predicting both gradient and counter-gradient behaviours of $F_{j}$. It has been found in previous analyses that Clark's gradient model (CGM) (Clark et al. 1979) for SGS scalar flux (Gao et al. 2015a, b; Klein et al. 2016, 2018c) provides satisfactory performance, which has been utilised here to propose an alternative model as:

$$
F_{j}=\left[\overline{\rho u_{j} c^{2}}-2\left[\overline{\rho u_{j} c}-\bar{\rho} \tilde{u_{j}} \tilde{c}\right] \tilde{c}-\bar{\rho} \tilde{u_{j}} \widetilde{c^{2}}\right]=\bar{\rho} \frac{\Delta^{2}}{12} \frac{\partial \tilde{u}_{j}}{\partial x_{k}} \frac{\partial \sigma_{v}^{2}}{\partial x_{k}}
$$

The predictions of $F_{j}^{C G M}$ are also shown in Fig. 5 which indicates that this model is capable of capturing both gradient and counter-gradient behaviours of SGS flux of variance. However, the quantitative agreement between DNS data and model prediction deteriorates with increasing $\Delta$.

A model expression, which was originally proposed by Chakraborty and Swaminathan (CSM) (2011) for the Reynolds flux of variance in the context of RANS, has been extended for LES in the present work in the following manner. According to Bray Moss Libby modelling (Bray et al. 1985) the Reynolds flux of variance can be expressed based on the presumed bi-modal PDF of $c$ with impulses at $c=0$ and $c=1.0$ as:

$$
\begin{gathered}
\left\langle\rho u_{i}^{\prime \prime} c^{\prime \prime 2}\right\rangle=\langle\rho\rangle \tilde{c}(1-\tilde{c})\left[\left\langle u_{i}\right\rangle_{P}-\left\langle u_{i}\right\rangle_{R}\right](1-2 \widetilde{c})+O\left(D a^{-1}\right) \\
=\left\langle\rho u_{i}^{\prime \prime} c^{\prime \prime}\right\rangle(1-2 \widetilde{c})+O\left(D a^{-1}\right)
\end{gathered}
$$
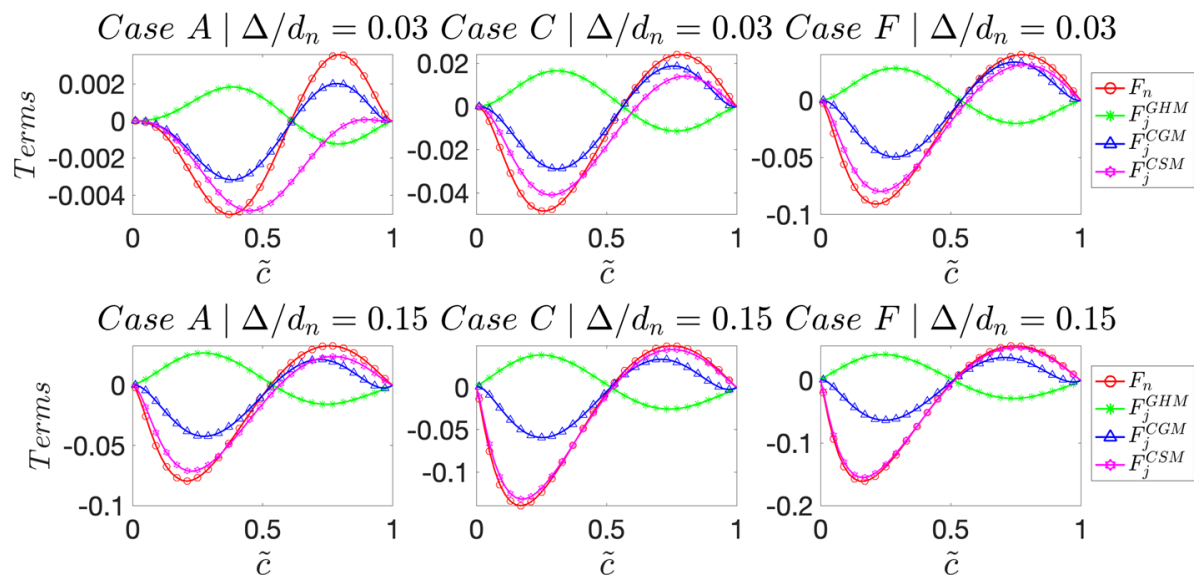

Fig. 5 Variations of mean values of $F_{n} / \rho_{0} S_{L}$ conditional upon bins of $\tilde{c}$ for $\Delta / d_{n}=0.03$ and 0.15 in cases A, C, F along with the predictions of $F_{j}^{G H M}$ (for $S c_{t}=1.0$ ), $F_{j}^{C G M}$ and $F_{j}^{C S M}$ 
where $\langle q\rangle$ and $\tilde{q}=\langle\rho q\rangle /\langle\rho\rangle$ are the Reynolds averaged and Favre-averaged values of a general quantity $q$, respectively and $\langle q\rangle_{P}$ and $\langle q\rangle_{R}$ are conditional mean values of the quantity $q$ in products and reactants, respectively. However, Eq. 8 does not perform well for $D a<1$ where the PDF of $c$ does not remain bi-modal (Bray et al. 1985). The departure from bimodal PDF can be quantified with the help of a segregation factor $g=\left\langle\rho c^{\prime \prime} c^{\prime \prime}\right\rangle /\left[\langle\rho\rangle^{m}(1-\tilde{c})\right]$ so that $g$ increasingly deviates from unity with decreasing Damköhler number $D a$. In order to extend Eq. 8 for $D a<1$ combustion, Chakraborty and Swaminathan (2011) proposed the following model expression and validated it with the help of a priori analysis:

$$
\left\langle\rho u_{i}^{\prime \prime} c^{\prime \prime 2}\right\rangle=\left\langle\rho u_{i}^{\prime \prime} c^{\prime \prime}\right\rangle\left(1-2 \widetilde{c} g^{m}\right)[2 g /(g+1)] ; m=0.3
$$

It can be seen from Eq. 9 that this expression reduces to Eq. 8 when $g=1.0$ which is likely to be realised for $D a \gg 1$ where the PDF of $c$ is likely to be bi-modal. In the context of LES, the model can be written as:

$$
F_{j}^{C S M}=\left[\overline{\rho u_{j} c}-\bar{\rho} \tilde{u_{j}} \tilde{c}\right]\left[1-2 \tilde{c}\left\{\sigma_{v}^{2} / \tilde{c}(1-\tilde{c})\right\}^{0.3}\right]\left\{2 \sigma_{v}^{2} /\left[\sigma_{v}^{2}+\tilde{c}(1-\tilde{c})\right]\right\}
$$

Figure 5 shows that both qualitative and quantitative predictions of $F_{n}$ can be obtained by using $F_{j}^{C S M}$ for all filter widths and cases considered here. The satisfactory performance of $F_{j}^{C S M}$ depends on appropriate modelling of the SGS scalar flux $\left[\overline{\rho u_{j} c}-\bar{\rho} \tilde{u_{j}} \tilde{c}\right]$, which has been addressed elsewhere (Gao et al. 2015a, b; Klein et al. 2016, 2018c) and thus is not repeated here.

The modelling of $T_{3}=2[\overline{\dot{w} c}-\overline{\dot{w}} \tilde{c}]$ needs closures of $\overline{\dot{w}}$ and $\overline{\dot{w} c}$. For unity Lewis number flames, it is possible to write: $\overline{\dot{w}}=\int_{0}^{1}[\dot{w}]_{L} P_{\Delta}(c) d c$ and $\overline{\dot{w} c}=\int_{0}^{1}[\dot{w} c]_{L} P_{\Delta}(c) d c$ (Bray 1980 ) where $[q]_{L}$ is the value of a general variable $q$ obtained from a flamelet table and $P_{\Delta}(c)$ is the presumed sub-grid PDF of $c$, which is usually parameterised using $\tilde{c}$ and $\sigma_{v}^{2}$ (Langella and Swaminathan 2016; Langella et al. 2016). Alternatively, it is possible to write: $\overline{\dot{w} c}=\overline{\dot{w}} c_{m}$ where $c_{m}=\int_{0}^{1}[\dot{w} c]_{L} f(c) d c / \int_{0}^{1}[\dot{w}]_{L} f(c) d c$ (Bray 1980), with $f(c)$ being the burning mode PDF, which can be taken as any appropriate continuous function according to Bray (Bray 1980). This thermo-chemical parameter $c_{m}$ has been found to be 0.84 for cases A-F. Accordingly, $T_{3}$ can be expressed as: $T_{3}=2 \overline{\dot{w}}\left[c_{m}-\tilde{c}\right]$. The variations of mean values of $T_{3}$ conditional upon bins of $\tilde{c}$ in cases $\mathrm{A}, \mathrm{C}, \mathrm{F}$ are shown in Fig. 6 for $\Delta / d_{n}=0.03$ and 0.15 , which shows that $2 \overline{\dot{w}}\left[c_{m}-\tilde{c}\right]$ (with $\overline{\dot{w}}$ extracted from DNS data) satisfactorily captures both qualitative and quantitative behaviours of $T_{3}$ for all $\Delta$ in all cases considered here. According to Bray (Bray 1980), the mean reaction rate in the context of RANS for $D a \gg 1$ can be modelled by: $\langle\dot{w}\rangle=2\left\langle\rho N_{c}\right\rangle /\left(2 c_{m}-1\right)$. It has been shown in Dunstan et al. (2013), Gao et al. (2014a) and Ma et al. (2014a) based on a priori analysis of DNS that the expression $\overline{\dot{w}}=\left\{2 \bar{\rho} \tilde{N}_{c} /\left(2 c_{m}-1\right)\right\}$ performs well for $\Delta / \delta_{t h} \gg 1$ and the agreement between $\overline{\dot{w}}$ and $\left\{2 \bar{\rho} \tilde{N}_{c} /\left(2 c_{m}-1\right)\right\}$ improves with increasing $\Delta$, as sub-grid Damköhler number $D a_{\Delta}=\Delta S_{L} / u_{\Delta}^{\prime} \delta_{t h}$ increases with an increase in LES filter width (see Fig. 3). However, $\left\{2 \bar{\rho} \tilde{N}_{c} /\left(2 c_{m}-1\right)\right\}$ does not adequately predict $\overline{\dot{w}}$ for small filter widths (i.e. $\Delta / \delta_{t h}<1$ ) when $D a_{\Delta}$ assumes small values. In order to obtain a single expression which predicts $\overline{\dot{w}}$ accurately for both small and large filter widths the following model expression was suggested in Dunstan et al. (2013), Gao et al. (2014a) and Ma et al. (2014a):

$$
\overline{\dot{w}}=\left\{2 \bar{\rho} \tilde{N}_{c} /\left(2 c_{m}-1\right)\right\} \times\left\{1-\exp \left(-\phi \Delta / \delta_{t h}\right)\right\}+f_{1}(\bar{\rho}, \tilde{c}, \tilde{T}) \exp \left(-\phi \Delta / \delta_{t h}\right)
$$




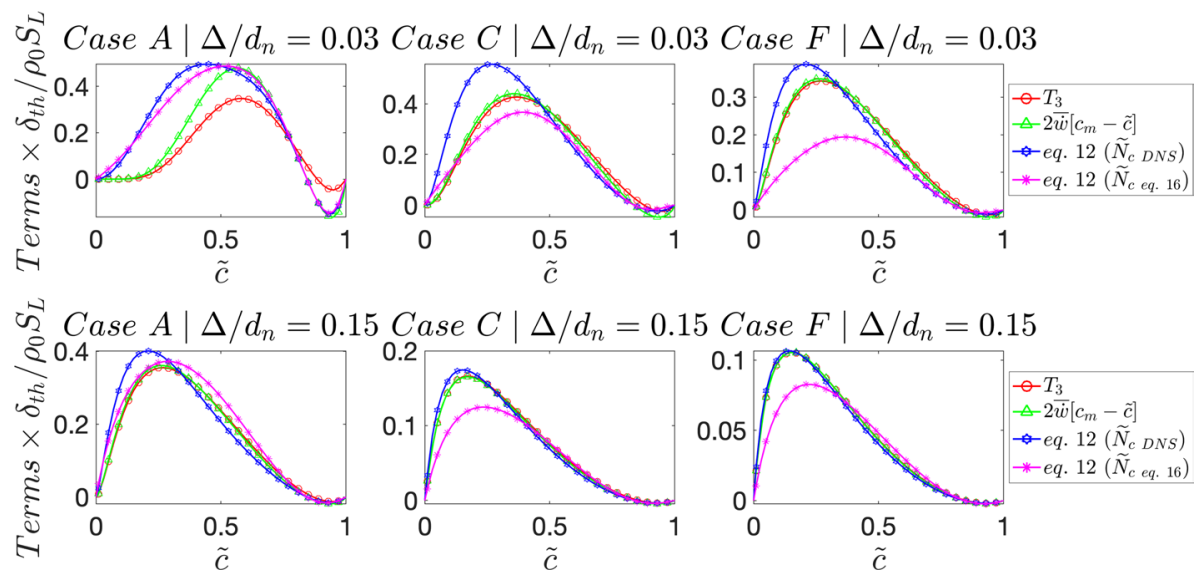

Fig. 6 Variations of mean values of $T_{3} \times \delta_{t h} / \rho_{0} S_{L}, 2 \overline{\dot{w}}\left[c_{m}-\tilde{c}\right] \times \delta_{t h} / \rho_{0} S_{L}$ and the predictions of Eq. 12 (for $\tilde{N}_{c}$ extracted from DNS data and according to the prediction of Eq. 16 with $\beta_{c}$ according to Eq. 18) conditional upon bins of $\tilde{c}$ for $\Delta / d_{n}=0.03$ and 0.15 in cases $\mathrm{A}, \mathrm{C}, \mathrm{F}$

where $f_{1}(\bar{\rho}, \tilde{c}, \tilde{T})$ is a function such that $\dot{w}=f_{1}(\rho, c, T)$ where $T$ is the temperature, and $\phi=0.56 \delta_{t h} S_{L} / \alpha_{T 0}$ is a model parameter with $\alpha_{T 0}$ being the thermal diffusivity in the unburned gas. The exponential terms $\exp \left(-\phi \Delta / \delta_{t h}\right)$ and $\left\{1-\exp \left(-\phi \Delta / \delta_{t h}\right)\right\}$ act as bridging function such that one obtains $\overline{\dot{w}}=\left\{2 \bar{\rho} \tilde{N}_{c} /\left(2 c_{m}-1\right)\right\}$ for $\Delta / \delta_{\text {th }} \gg 1$, and for $\Delta \rightarrow 0$, Eq. 11 reduces to $\lim _{\Delta \rightarrow 0} \overline{\dot{w}}=\dot{w}=\lim _{\Delta \rightarrow 0} f_{1}(\bar{\rho}, \tilde{c}, \tilde{T})=f_{1}(\rho, c, T)$. It has been shown elsewhere (Gao et al. 2014a; Ma et al. 2014a) that the prediction of Eq. 11 shows better agreement with $\overline{\dot{w}}$ extracted from DNS data only for $\Delta>\delta_{t h}$ and the quantitative agreement improves with increasing filter width. Using Eq. 11 in $T_{3}=2[\overline{\dot{w} c}-\overline{\dot{w}} \tilde{c}]=2 \overline{\dot{w}}\left\{c_{m}-\tilde{c}\right\}$ yields:

$$
T_{3}=2\left[2 \bar{\rho} \tilde{N}_{c} /\left(2 c_{m}-1\right)\left\{1-\exp \left(-\phi \Delta / \delta_{t h}\right)\right\}+f_{1}(\bar{\rho}, \tilde{c}, \tilde{T}) \exp \left(-\phi \Delta / \delta_{t h}\right)\right]\left(c_{m}-\tilde{c}\right)
$$

Figure 6 shows that $T_{3}$ can be reasonably predicted by this expression and the quantitative agreement improves with increasing $\Delta$. This behaviour arises due to improved prediction of $\overline{\dot{w}}$ by Eq. 11 for large values of $\Delta$ (e.g. $\Delta=0.15 d_{n}$ ) (Dunstan et al. 2013; Gao et al. 2014a; Ma et al. 2014a).

The transport equation of $\tilde{N}_{c}$ takes the following form (Gao et al. 2014a, 2015c; Chakraborty et al. 2011):

$$
\frac{\partial\left(\bar{\rho} \tilde{N}_{c}\right)}{\partial t}+\frac{\partial\left(\bar{\rho} \tilde{u}_{j} \tilde{N}_{c}\right)}{\partial x_{j}}=\underbrace{\overline{\frac{\partial}{\partial x_{j}}\left(\rho D \frac{\partial N_{c}}{\partial x_{j}}\right)}}_{D_{1}}+T_{1}^{\prime}+T_{2}^{\prime}+T_{3}^{\prime}+T_{4}^{\prime}-D_{2}+f(D)
$$

where $u_{j}$ is the jth component of velocity vector and the terms on the left-hand side denote the transient effects and resolved advection of $\tilde{N}_{c}$ respectively. The term $D_{1}=\overline{\nabla \cdot\left(\rho D \nabla N_{c}\right)}$ represents the molecular diffusion of $\tilde{N}_{c}$ and the terms $T_{1}^{\prime}, T_{2}^{\prime}, T_{3}^{\prime}, T_{4}^{\prime},\left(-D_{2}\right)$ and $f(D)$ are unclosed and given by: 


$$
\begin{aligned}
& T_{1}^{\prime}=-\frac{\partial}{\partial x_{j}}\left[\overline{\rho u_{j} N_{c}}-\bar{\rho} \tilde{u}_{j} \tilde{N}_{c}\right] \\
& T_{2}^{\prime}=-\overline{\frac{2 D}{\rho}\left[\dot{w}+\frac{\partial}{\partial x_{j}}\left(\rho D \frac{\partial c}{\partial x_{j}}\right)\right] \frac{\partial c}{\partial x_{k}} \frac{\partial \rho}{\partial x_{k}}} \\
& T_{3}^{\prime}=-2 \rho \overline{\frac{\partial c}{\partial x_{i}} \frac{\partial u_{i}}{\partial x_{j}} \frac{\partial c}{\partial x_{j}}} \\
& T_{4}^{\prime}=2 \overline{D \frac{\partial \dot{w}}{\partial x_{i}} \frac{\partial c}{\partial x_{i}}} \\
& \left(-D_{2}\right)=-2 \rho \overline{D^{2} \frac{\partial^{2} c}{\partial x_{i} \partial x_{j}} \frac{\partial^{2} c}{\partial x_{i} \partial x_{j}}} \\
& f(D)=\overline{2 D \frac{\partial c}{\partial x_{k}} \frac{\partial(\rho D)}{\partial x_{k}} \frac{\partial^{2} c}{\partial x_{j} \partial x_{j}}}+\overline{2 D \frac{\partial c}{\partial x_{k}} \frac{\partial^{2}(\rho D)}{\partial x_{j} \partial x_{k}} \frac{\partial c}{\partial x_{j}}}-\overline{\frac{\partial}{\partial x_{j}}\left(\rho N_{c} \frac{\partial D}{\partial x_{j}}\right)} \\
& -\overline{-2 \rho D \frac{\partial D}{\partial x_{j}} \frac{\partial}{\partial x_{j}}\left(\frac{\partial c}{\partial x_{k}} \frac{\partial c}{\partial x_{k}}\right)}+\overline{\rho\left(\frac{\partial c}{\partial x_{k}} \frac{\partial c}{\partial x_{k}}\right)\left[\frac{\partial D}{\partial t}+u_{j} \frac{\partial D}{\partial x_{j}}\right]}
\end{aligned}
$$

The term $T_{1}^{\prime}$ represents the effects of sub-grid convection, whereas $T_{2}^{\prime}$ denotes the effects of density-variation due to heat release. The term $T_{3}^{\prime}$ is determined by the alignment of $\nabla c$ with local strain rates $e_{i j}=0.5\left(\partial u_{i} / \partial x_{j}+\partial u_{j} / \partial x_{i}\right)$ and this term is commonly referred to as the scalar-turbulence interaction term. The term $T_{4}^{\prime}$ arises due to the correlation between $\nabla \dot{w}$ and $\nabla c$, whereas $\left(-D_{2}\right)$ denotes the molecular dissipation of scalar dissipation rate and these terms will henceforth be referred to as the reaction rate term and dissipation term respectively. The term $f(D)$ denotes the effects of $D$ variation. It has been demonstrated based on scaling arguments by Gao et al. (2014b) that an equilibrium is maintained between the $\left(T_{2}^{\prime}+T_{3}^{\prime}+T_{4}^{\prime}+f(D)\right)$ and $\left(-D_{2}\right)$ for large filter widths (i.e. $\left.\Delta / \delta_{t h} \gg 1\right)$. A similar conclusion can be reached if the transport equation of $\widetilde{\varepsilon}_{c}=\langle\rho D \nabla c \cdot \nabla c\rangle /\langle\rho\rangle-\tilde{D} \nabla \tilde{c} \cdot \nabla \tilde{c}$ is analysed and the terms equivalent to $\left(T_{2}^{\prime}+T_{3}^{\prime}+T_{4}^{\prime}+f(D)\right)$ and $\left(-D_{2}\right)$ are considered (Chakraborty et al. 2008). Kolla et al. (2009) and Chakraborty and Swaminathan (2011) considered the modelled expressions for the terms equivalent to $T_{2}^{\prime}, T_{3}^{\prime},\left(T_{4}^{\prime}-D_{2}+f(D)\right)$ in the context of RANS and utilised their balance (i.e. $\left(T_{2}^{\prime}+T_{3}^{\prime}+T_{4}^{\prime}+f(D)-D_{2}\right) \approx 0$ ) to propose a model expression:

$$
\stackrel{m}{\varepsilon}_{c}=\left[2 K_{c}^{*} S_{L} / \delta_{t h}+\left(C_{3}^{*}-\tau D a_{\Delta} C_{4}^{*}\right) \stackrel{m}{\varepsilon} / \tilde{k}\right] \tilde{c}(1-\tilde{c}) / \beta_{c}{ }^{\prime} ; \beta_{c}^{\prime}=6.7
$$

where $C_{3}^{*}, C_{4}^{*}$ and $\beta_{c}^{\prime}$ are the model parameters and $K_{c}^{*}=\left(\delta_{t h} / S_{L}\right) \int_{0}^{1}\left[\rho N_{c} \nabla \cdot \vec{u}\right]_{L}$ $f(c) d c / \int_{0}^{1}\left[\rho N_{c}\right]_{L} f(c) d c$ is a thermo-chemical parameter (Chakraborty and Swaminathan 2011). Equation 15 has been extended to LES based on the equilibrium between the $\left(T_{2}^{\prime}+T_{3}^{\prime}+T_{4}^{\prime}+f(D)\right)$ and $\left(-D_{2}\right)$ for large filter widths (i.e. $\Delta / \delta_{t h} \gg 1$ ) in the following manner (Dunstan et al. 2013; Gao et al. 2014a; Ma et al. 2014a): 


$$
\tilde{N}_{\mathrm{c}}=\tilde{D} \nabla \tilde{c} \cdot \nabla \tilde{c}+\left(1-f_{b}\right)\left[2 K_{c}^{*} S_{L} / \delta_{t h}+\left(C_{3}^{*}-\tau D a_{\Delta} C_{4}^{*}\right) 2 u_{\Delta}^{\prime} / 3 \Delta\right] \tilde{c}(1-\tilde{c}) / \beta_{c}
$$

The model parameters $C_{3}^{*}, C_{4}^{*}$ and $\beta_{c}$ are given by the following expressions (Gao et al. 2014a; Ma et al. 2014a):

$$
\begin{aligned}
& C_{3}^{*}=\frac{2.0 \sqrt{K a_{\Delta}}}{1.0+\sqrt{K a_{\Delta}}} ; \quad C_{4}^{*}=\frac{1.2(1.0-\tilde{c})^{0.2}}{L e^{2.57}\left(1+K a_{\Delta}\right)^{0.4}} ; \\
& \beta_{c}=\max \left(\frac{2}{2 c_{m}-1},\left[1.05 \frac{\tau}{\tau+1}+0.51\right]^{4.6}\right)
\end{aligned}
$$

where $K a_{\Delta}=\left(u_{\Delta}^{\prime} / S_{L}\right)^{3 / 2}\left(\Delta / \delta_{t h}\right)^{-1 / 2}$ is the sub-grid Karlovitz number. It is worth noting that $\beta_{c}$ in LES is different from $\beta_{c}^{\prime}$ used in the context of RANS (Chakraborty and Swaminathan 2011; Kolla et al. 2009). The model parameter $\beta_{c}$ (and $\beta_{c}^{\prime}$ ) originate from the model expression of $\left(T_{4}^{\prime}-D_{2}+f(D)\right)$. The combined contribution of the terms $D_{1}, T_{4}^{\prime},\left(-D_{2}\right)$ and $f(D)$ can be written as (Chakraborty et al. 2008, 2011; Gao and Chakraborty 2016):

$$
D_{1}+T_{4}^{\prime}-D_{2}+f(D) \approx-\overline{D \nabla \nabla \cdot\left(\rho S_{d} \vec{N}|\nabla c|\right)|\nabla c|}+2 \overline{\rho D S_{d} \nabla \cdot \vec{N}|\nabla c|^{2}}
$$

where $S_{d}=[\dot{w}+\nabla \cdot(\rho D \nabla c)] /(\rho|\nabla c|)$ is the displacement speed. It can be seen with Eq. 17 that the net contribution of $\left[D_{1}+T_{4}^{\prime}-D_{2}+f(D)\right]$ depends on flame curvature $\kappa_{m}=0.5 \nabla \cdot \vec{N}$ (with $\vec{N}=-\nabla c /|\nabla c|$ being the flame normal vector). This suggests that the model parameter $\beta_{c}$ (and $\beta_{c}^{\prime}$ ) is likely to be dependent on flame curvature description in the underlying computational methodology. As flame wrinkling is partially resolved in LES in contrast to the total sub-grid curvature contribution in RANS, the modification from $\beta_{c}^{\prime}$ to $\beta_{c}$ in the model expression of scalar dissipation rate is not completely unexpected. It is important to note that the model parameters $C_{3}^{*}, C_{4}^{*}$ and $\beta_{c}$ have been calibrated using a large number of DNS cases including variations of $\tau, L e, D a$ and $K a$ (see Gao et al. 2014a) in the past, and thus, the dependence of $\mathrm{Ka}$ is explicitly addressed.

In Eq. 16i (Dunstan et al. 2013; Gao et al. 2014a; Ma et al. 2014a), $K_{c}^{*}=\left(\delta_{t h} / S_{L}\right) \int_{0}^{1}\left[\rho N_{c} \nabla \cdot \vec{u}\right]_{L} f(c) d c / \int_{0}^{1}\left[\rho N_{c}\right]_{L} f(c) d c$ is a thermo-chemical parameter (Chakraborty and Swaminathan 2011), which is found to be $K_{c}^{*}=0.77 \tau$ for cases A-F for the present thermo-chemistry and $f_{b}=\exp \left[-0.7\left(\Delta / \delta_{t h}\right)^{1.7}\right]$ is a bridging function, that ensures that $\tilde{N}_{c}$ approaches $D \nabla c \cdot \nabla c$, when the flame is fully resolved (i.e. $\lim _{\Delta \rightarrow 0} \tilde{N}_{c}=D \nabla c \cdot \nabla c$ ). The model parameters $C_{3}^{*}, C_{4}^{*}$ and $\beta_{c}$ are given by Eq. 16 ii (Gao et al. 2014a; Ma et al. 2014a), where $K a_{\Delta}=\left(u_{\Delta}^{\prime} / S_{L}\right)^{3 / 2}\left(\Delta / \delta_{t h}\right)^{-1 / 2}$ is the sub-grid Karlovitz number. The predictions of Eq. 16i are compared to the mean values of $\tilde{N}_{c}$ extracted from DNS conditional upon the bins of $\tilde{c}$ in Fig. 7 for $\Delta / d_{n}=0.03$ and 0.15 , which shows that this model satisfactorily predicts $\tilde{N}_{c}$ for small filter widths (e.g. $\Delta / d_{n}=0.03$ ) where the resolved component (i.e. $\tilde{D} \nabla \tilde{c} \cdot \nabla \tilde{c}$ ) plays the dominant role but over predictions can be observed for large filter widths (e.g. $\Delta / d_{n}=0.15$ ). This is consistent with previous findings (Gao et al. 2014a; Ma et al. 2014a), which also showed that Eq. 16i overpredicts the mean values of $\tilde{N}_{c}$ conditional upon $\tilde{c}$ for small values of $u^{\prime} / S_{L}$ but this overprediction disappears for large values of $u^{\prime} / S_{L}$. The extent of this overprediction is particularly severe for large filter widths (e.g. $\Delta / d_{n}=0.15$ ) for high pressure cases $\mathrm{B}, \mathrm{C}$ and $\mathrm{F}$ and the extent of overprediction increases with increasing pressure.

A given value of $\Delta / d_{n}$ implies larger value of $\Delta / \delta_{t h}$ for higher pressures as the flame thickness $\delta_{t h}$ decreases with increasing pressure. The overprediction of the mean values of 
$\tilde{N}_{c}$ conditional upon $\tilde{c}$ according to Eq. $16 \mathrm{i}$ in cases $\mathrm{B}, \mathrm{C}$ and $\mathrm{F}$ has been found to be comparable to that in cases $\mathrm{A}, \mathrm{D}$ and $\mathrm{E}$ for a given value of $\Delta / \delta_{t h}$ (not shown here).

It has been shown elsewhere (Chakraborty and Swaminathan 2011; Chakraborty et al. 2008, 2011; Gao and Chakraborty 2016; Gao et al. 2016) that the model parameter $\beta_{c}$ originates due to the curvature $\kappa_{m}$ contribution to the SDR transport and $\kappa_{m}$ dependence of $S_{d}$ plays a key role in this contribution. It has been shown and discussed elsewhere (Klein et al. 2018b; Alquallaf et al. 2019) that the flame curvature statistics are significantly affected by pressure. The likelihood of obtaining DL instability increases with increasing pressure (Klein et al. 2018a, b; Alquallaf et al. 2019) and this is reflected in the increased skewness of negative curvature values in cases B, C and F (not shown here but refer to Klein et al. 2018a, b; Alquallaf et al. 2019). Thus, some pressure dependence of $\beta_{c}$ is not unexpected. It has been found that the value of $\beta_{c}$, which ensures that the volume-integrated $\bar{\rho} \tilde{N}_{c}$ obtained from DNS data can be satisfactorily predicted, needs to increase with increasing pressure. This has been empirically parameterised as:

$$
\beta_{c}=\max \left(2 /\left(2 c_{m}-1\right),\left(p / p_{0}\right)^{0.37}[1.05 \tau /(\tau+1)+0.51]^{4.6}\right)
$$

It can be seen from Fig. 7 that the use of $\beta_{c}$ expression given by Eq. 18 in Eq. 16 provides better agreement with DNS data. Furthermore, Fig. 7 shows that using Eq. 16i with $\beta_{c}$ given by Eq. 18 in Eq. 12 provides satisfactory prediction of $T_{3}$ in all cases. It is worth noting that $T_{3}$ is overpredicted if Eq. 16i is used without the pressure dependence of $\beta_{c}$ and thus is not explicitly shown here. Finally, as $\tilde{D} \nabla \tilde{c} \cdot \nabla \tilde{c}$ is a resolved quantity, Eq. $16 \mathrm{i}$ with $\beta_{c}$ according to Eq. 18 can be used to close the molecular dissipation term $D_{v}=-\bar{\rho} \tilde{\varepsilon}_{c}=-\bar{\rho}\left[\tilde{N}_{c}-\tilde{D} \nabla \tilde{c} \cdot \nabla \tilde{c}\right]$.

In the present analysis all statistics are taken from the whole flame. This is based on the finding that there is little variation between different axial locations for a variety of quantities (Klein et al. 2018d). Finally, it is worth noting that the models discussed in this paper need to be implemented in actual LES simulations because numerical and modelling errors interact in a complex manner so the applicability of these models cannot entirely be assessed based on a priori DNS analysis. Interested authors are referred to (Vervisch et al. 2008) for discussion on the coupling between the numerical discretization of scalar field transport and the modelling of unresolved sub-grid scale fluctuations of chemical species. The novelty of the current analysis lies in the analysis of transport equation-based closure of SGS scalar variance for turbulent premixed flames under elevated pressures, which is yet to be carried out in the existing literature. Therefore, model capabilities are a priori assessed in this analysis without the interference
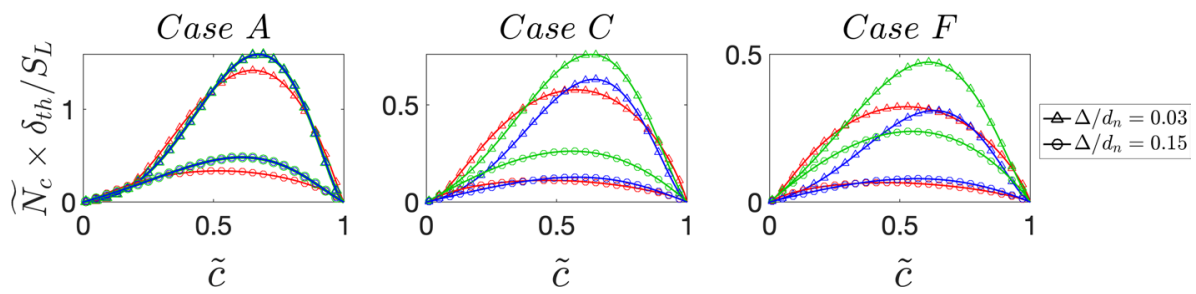

Fig. 7 Variations of mean values of $\tilde{N}_{c} \times \delta_{t h} / S_{L}$ (red) and the predictions of Eq. 16i with $\beta_{c}$ given by Eq. 16ii (green) and $\beta_{c}$ according to Eq. 18 (blue) conditional upon bins of $\tilde{c}$ for $\Delta / d_{n}=0.03$ and 0.15 in cases A, C, F 
of numerical errors because a posteriori assessment of models using LES is intrinsically code dependent as a result of the interaction of numerical and modelling errors (Vervisch et al. 2008). It is worth noting that the same approach has been adopted in several previous studies (Ranjan et al. 2016; Nilsson et al. 2019; Reddy and Abraham 2012; Veynante et al. 1997; Charlette et al. 2002a; Selle and Bellan 2007; Lignell et al. 2009; Chatakonda et al. 2013; Lapointe and Blanquart 2017; Aspden et al. 2019; Devaud et al. 2019; Bushe et al. 2020) by other authors and there are several examples where the models proposed based on a priori DNS analysis (e.g. Gao et al. 2014a; Charlette et al. 2002a) have been demonstrated to perform well based on a posteriori assessment (e.g. Charlette et al. 2002b; Ma et al. 2013, 2014a, b; Butz et al. 2015). A recent analysis by different authors (Nilsson et al. 2019) on the modelling of SGS variance transport has also been conducted entirely by a priori assessment and the same approach has been adopted in the current study.

It is also worth mentioning that SGS variance transport has been adopted in several LES simulations (Langella and Swaminathan 2016; Langella et al. 2016, 2018; Massey et al. 2018; Chen et al. 2019a; b, 2020; Massey et al. 2019) in the past for atmospheric flames, and these simulations successfully used several model expressions considered in this analysis (e.g. Eqs. 6, 16i and 16ii) for closures of SGS flux of variance and SDR. The modelling of the chemical reaction contribution $T_{3}$ is reliant upon the SDR based filtered reaction rate closure given by Eq. 11. This SDR based reaction rate closure was also previously successfully implemented in LES albeit for atmospheric premixed turbulent combustion (Ma et al. 2014a; Butz et al. 2015) and the SDR closure given by Eqs. 16i and 16ii has been used in several previous LES studies (Ma et al. 2014a; Butz et al. 2015; Langella et al. 2018; Massey et al. 2018; Chen et al. 2019a, b, 2020; Massey et al. 2019) for atmospheric premixed combustion. The current analysis justifies the choices of the model expressions given by Eqs. 11, 16i and 16ii based on a priori DNS analysis. The only two expressions which are yet to be a posteriori analysed by implementing them in LES are the SGS variance flux model given by Eq. 10 and the pressure correction in the SDR modelling given by Eq. 18. A close inspection of Eq. 10 reveals that the success of this model depends on the successful modelling of SGS flux of reaction progress variable $\left[\overline{\rho u_{j} c}-\bar{\rho} \tilde{u_{j}} \tilde{c}\right]$ and the modelling of $\left[\overline{\rho u_{j} c}-\bar{\rho} \tilde{u_{j}} \tilde{c}\right]$ has been addressed elsewhere (Allauddin et al. 2017) by simultaneous a priori DNS and a posteriori LES analyses.

It is important to note that an experimental database, which offers information regarding SGS variance, and its sub-grid flux and dissipation rate for a range of elevated pressure conditions, is rare in the existing literature. Thus, a posteriori assessment of the models of the unclosed terms in the SGS variance transport equation is not a straightforward task because a discrepancy between LES results and experimental measurements may not originate from the inaccuracy in the SGS variance modelling and similarly a good agreement between LES and experimental results might be obtained due to fortuitous cancellation of errors. However, notwithstanding the above comments, the model expressions identified in this analysis need to be implemented in LES for an experimental configuration for which measurements are available for the assessment of the performances of the SGS variance closure. 


\section{Conclusions}

The modelling of SGS variance of reaction progress variable and its sensitivity to thermodynamic pressure have been analysed based on a priori analysis of DNS data of turbulent premixed Bunsen burner flames at different pressure levels. An algebraic expression of the SGS variance based on the presumed bi-modal distribution with impulses at $c=0.0$ and 1.0 has been found to be inadequate even for the flames in the corrugated/ wrinkled flamelets regime. An alternative algebraic expression of SGS variance, which is usually used for passive scalar mixing, has been shown to significantly overpredict the corresponding quantity extracted from DNS data. The statistical behaviours of the unclosed terms of the SGS variance transport equation have been analysed. It has been found that the reaction rate contribution plays a leading order role for all filter widths and remains of the same order as that of the molecular dissipation contribution for large filter widths. The modelling of the unclosed terms of the SGS variance transport equation has been assessed based on a priori DNS analysis and suitable models have been identified for the SGS flux of variance, reaction rate contribution and scalar dissipation rate. One of the model parameters of a widely used algebraic closure of the Favrefiltered SDR closure (Dunstan et al. 2013; Gao et al. 2014a; Ma et al. 2014a) has been found to be dependent on pressure, whereas the model performance for the SGS flux of variance has been found to be independent of pressure variation. As the effects of Darrieus-Landau instability are likely to become weak for high values of $K a$, the modelling methodology needs to be assessed for high pressure flames under high Karlovitz number. Moreover, these model expressions need to be implemented in actual LES for a posteriori assessment for comprehensive validation, which will form the basis of future analyses.

Acknowledgements Open Access funding provided by Projekt DEAL. The authors are grateful to EPSRC (EP/R029369/1) and the German Research Foundation (DFG, KL1456/5-1) for financial assistance, and ARCHER, Rocket-HPC (Newcastle University) and Gauss Centre for Supercomputing (Grant: pn69ga) for computational support.

\section{Compliance with Ethical Standards}

Conflict of interest We have no competing interests.

Ethics Statement This work did not involve any active collection of human data.

Open Access This article is licensed under a Creative Commons Attribution 4.0 International License, which permits use, sharing, adaptation, distribution and reproduction in any medium or format, as long as you give appropriate credit to the original author(s) and the source, provide a link to the Creative Commons licence, and indicate if changes were made. The images or other third party material in this article are included in the article's Creative Commons licence, unless indicated otherwise in a credit line to the material. If material is not included in the article's Creative Commons licence and your intended use is not permitted by statutory regulation or exceeds the permitted use, you will need to obtain permission directly from the copyright holder. To view a copy of this licence, visit http://creativecommons.org/licenses/by/4.0/. 


\section{Appendix}

The mean behaviors of $\left\{T_{1}, T_{2}, T_{3}, T_{4}\right.$ and $\left.D_{v}\right\} \times \delta_{t h} / \rho_{0} S_{L}$ conditional upon bins of $\tilde{c}$ for $\Delta / \delta_{\text {th }}=0.8$ and 4.0 in cases A, C, F are shown in Fig. 8.
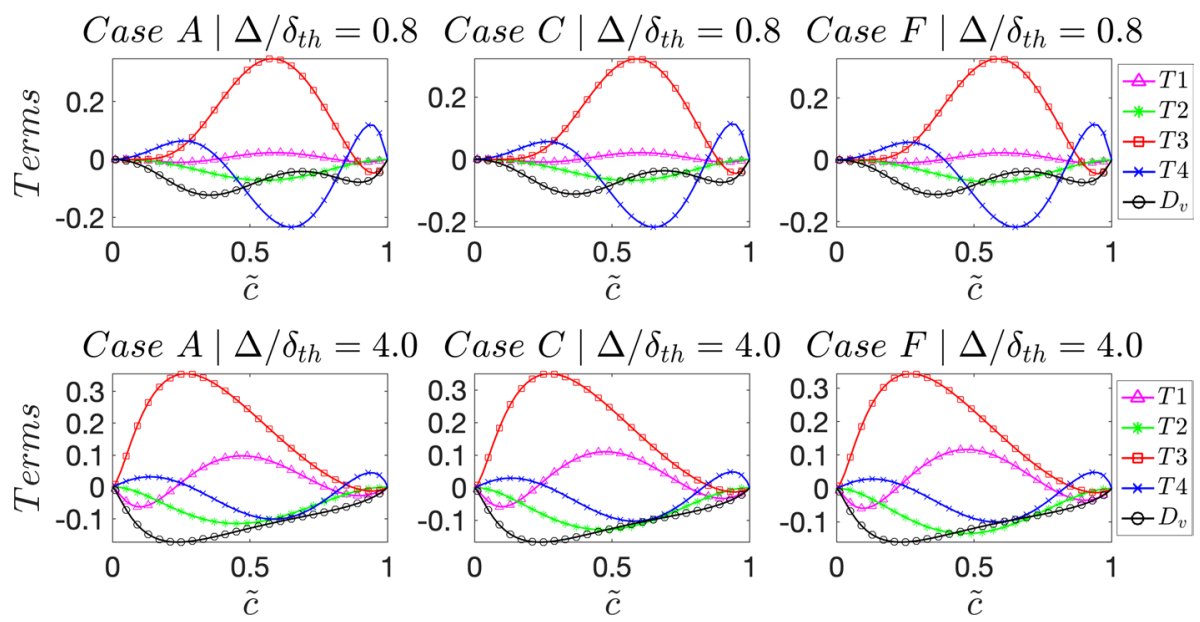

Fig. 8 Variations of mean values of $\left\{T_{1}, T_{2}, T_{3}, T_{4}\right.$ and $\left.D_{v}\right\} \times \delta_{t h} / \rho_{0} S_{L}$ conditional upon bins of $\tilde{c}$ for $\Delta / \delta_{t h}=0.8$ and 4.0 for cases $\mathrm{A}, \mathrm{C}, \mathrm{F}$

\section{References}

Allauddin, U., Pfitzner, M., Klein, M., Chakraborty, N.: A-priori and a posteriori analysis of algebraic flame surface density modelling in the context of large eddy simulation of turbulent premixed combustion. Num. Heat Transf. A. 71, 153-171 (2017)

Alquallaf, A., Klein, M., Dopazo, C., Chakraborty, N.: Evolution of flame curvature in turbulent premixed Bunsen flames at different pressure levels. Flow Turb. Combust. (2019). https://doi. org/10.1007/s10494-019-00027-X

Aspden, A.J., Zettervall, N., Fureby, C.: An a priori analysis of a DNS database of turbulent lean premixed methane flames for LES with finite-rate chemistry. Proc. Combust. Inst. 37, 2601-2609 (2019)

Boger, M., Veynante, D., Boughanem, H., Trouvé, A.: Direct numerical simulation analysis of flame surface density concept for large eddy simulation of turbulent premixed combustion. Proc. Combust. Inst. 27, 917-925 (1998)

Bray, K.N.C.: Turbulent flows with premixed reactants. In: Libby, P.A., Williams, F.A. (eds.) Turbulent Reacting Flows, pp. 115-183. Springer, Berlin (1980)

Bray, K.N.C., Libby, P.A., Moss, J.B.: Unified modeling approach for premixed turbulent combustion-part I: general formulation. Combust. Flame 61, 87-102 (1985)

Bushe, W.K., Devaud, C., Bellan, J.: A priori evaluation of the double-conditioned conditional source-term estimation model for high-pressure heptane turbulent combustion using DNS data obtained with onestep chemistry. Combust. Flame 217, 131-151 (2020)

Butz, D., Gao, Y., Kempf, A.M., Chakraborty, N.: Large Eddy Simulations of a turbulent premixed swirl flame using an algebraic scalar dissipation rate closure. Combust. Flame 162, 3180-3196 (2015)

Chakraborty, N., Cant, R.S.: A priori analysis of the curvature and propagation terms of the flame surface density transport equation for large eddy simulation. Phys. Fluids 19, 105101 (2007)

Chakraborty, N., Cant, R.S.: Direct numerical simulation analysis of the flame surface density transport equation in the context of large eddy simulation. Proc. Combust. Inst. 32, 1445-1453 (2009) 
Chakraborty, N., Swaminathan, N.: Effects of Lewis number on scalar variance transport in premixed flames. Flow Turb. Combust. 87, 261-292 (2011)

Chakraborty, N., Swaminathan, N.: Effects of turbulent Reynolds number on the scalar dissipation rate transport in turbulent premixed flames in the context of Reynolds averaged Navier Stokes simulations. Combust. Sci. Technol. 185, 676-709 (2013)

Chakraborty, N., Rogerson, J.W., Swaminathan, N.: A-Priori assessment of closures for scalar dissipation rate transport in turbulent premixed flames using direct numerical simulation. Phys. Fluids 20(045106), 1-15 (2008)

Chakraborty, N., Champion, M., Mura, A., Swaminathan, N.: Scalar dissipation rate approach to reaction rate closure. In: Swaminathan, N., Bray, K.N.C. (eds.) Turbulent Premixed Flame, 1st edn, pp. 74-102. Cambridge University Press, Cambridge (2011)

Chakraborty, N., Alwazzan, D., Klein, M., Cant, R.S.: On the validity of Damköhler's first hypothesis in turbulent Bunsen burner flames: a computational analysis. Proc. Combust. Inst. 37, 2231-2239 (2019)

Charlette, F., Meneveau, C., Veynante, D.: A power law wrinkling model for LES of premixed turbulent combustion, part I: non dynamic formulation and initial tests. Combust. Flame 131, 159-180 (2002a)

Charlette, F., Meneveau, C., Veynante, D.: A power law wrinkling model for LES of premixed turbulent combustion, part II: dynamic formulation. Combust. Flame 131, 181-197 (2002b)

Chatakonda, O., Hawkes, E.R., Aspden, A.J., Kerstein, A.R., Kolla, H., Chen, J.H.: On the fractal characteristics of low Damköhler number flames. Combust. Flame 160, 2422-2433 (2013)

Chen, Z., Swaminathan, N., Stöhr, M., Meier, W.: Interaction between self-excited oscillations and fuelair mixing in a dual swirl combustor. Proc. Combust. Inst. 37, 2325-2333 (2019a)

Chen, Z.X., Langella, I., Swaminathan, N., Stöhr, M., Meier, W., Kolla, H.: Large Eddy Simulation of a dual swirl gas turbine combustor: flame/flow structures and stabilisation under thermoacoustically stable and unstable conditions. Combust. Flame 203, 279-300 (2019b)

Chen, Z.X., Langella, I., Barlow, R.S., Swaminathan, N.: Prediction of local extinctions in piloted jet flames with inhomogeneous inlets using unstrained flamelets. Combust. Flame 212, 415-432 (2020)

Clark, R.A., Ferziger, J.H., Reynolds, W.C.: Evaluation of subgrid-scale models using an accurately simulated turbulent flow. J. Fluid Mech. 91, 1-16 (1979)

Devaud, C., Bushe, W.K., Bellan, J.: The modeling of the turbulent reaction rate under high-pressure conditions: a priori evaluation of the conditional source-term estimation concept. Combust. Flame 207, 205-221 (2019)

Dunstan, T., Minamoto, Y., Chakraborty, N., Swaminathan, N.: Scalar dissipation rate modelling for large eddy simulation of turbulent premixed flames. Proc. Combust. Inst. 34, 1193-1201 (2013)

Gao, Y., Chakraborty, N.: Modelling of Lewis number dependence of Scalar dissipation rate transport for Large Eddy Simulations of turbulent premixed combustion. Num. Heat Transf. A 69, 12011222 (2016)

Gao, Y., Chakraborty, N., Swaminathan, N.: Algebraic closure of scalar dissipation rate for large eddy simulations of turbulent premixed combustion. Combust. Sci. Technol. 186, 1309-1337 (2014a)

Gao, Y., Chakraborty, N., Swaminathan, N.: Scalar dissipation rate transport in the context of Large Eddy Simulations for turbulent premixed flames with non-unity Lewis number. Flow Turb. Combust. 93, 461-486 (2014b)

Gao, Y., Chakraborty, N., Klein, M.: Assessment of the performances of sub-grid scalar flux models for premixed flames with different global Lewis numbers: a direct numerical simulation analysis. Int. J. Heat Fluid Flow 52, 28-39 (2015a)

Gao, Y., Chakraborty, N., Klein, M.: Assessment of sub-grid scalar flux modelling in premixed flames for Large Eddy Simulations: a priori direct numerical simulation analysis. Eur. J. Mech. Fluids B 52, 97-108 (2015b)

Gao, Y., Chakraborty, N., Swaminathan, N.: Scalar dissipation rate transport and its modeling for large eddy simulations of turbulent premixed combustion. Combust. Sci. Technol. 187, 362-383 (2015c)

Gao, Y., Minamoto, Y., Tanahashi, M., Chakraborty, N.: A priori assessment of scalar dissipation rate closure for Large Eddy Simulations of turbulent premixed combustion using a detailed chemistry direct numerical simulation database. Combust. Sci. Technol. 188, 1398-1423 (2016)

Jenkins, K.W., Cant, R.S.: DNS of turbulent flame kernels. In: Liu, C., Sakell, L., Beautner, T. (eds.) Proceedings of 2nd AFOSR Conference on DNS and LES, pp. 192-202. Kluwer, Amsterdam (1999)

Jimenez, C., Ducros, F., Cuenot, B., Bedat, B.: Subgrid scale variance and dissipation of a scalar field in large eddy simulations. Phys. Fluids 13, 1748-1754 (2001) 
Keil, F.B., Chakraborty, N., Klein, M.: Sub-grid reaction progress variable close in turbulent premixed flames. In: Proceedings of 13th Medicine Combustion Symposium, 16th-20th June, Tenerife, Spain (2019)

Klein, M., Sadiki, A., Janicka, J.: A digital filter based generation of inflow data for spatially developing direct numerical or large eddy simulations. J. Comput. Phys. 186, 652-665 (2003)

Klein, M., Chakraborty, N., Gao, Y.: Scale similarity based models and their application to subgrid scale scalar flux modelling in the context of turbulent premixed flames. Int. J. Heat Fluid Flow 57, 91-108 (2016)

Klein, M., Alwazzan, D., Chakraborty, N.: A direct numerical simulation analysis of pressure variation in turbulent premixed Bunsen burner flames-part 1: scalar gradient and strain rate statistics. Comput. Fluids 173, 178-188 (2018a)

Klein, M., Nachtigal, H., Hansinger, M., Pfitzner, M., Chakraborty, N.: Flame curvature distribution in high pressure turbulent Bunsen premixed flames. Flow Turb. Combust. 101, 1173-1187 (2018b)

Klein, M., Kasten, C., Chakraborty, N., Mukhadiyev, N., Im, H.G.: Turbulent scalar fluxes in $\mathrm{H}_{2}$-air premixed flames at low and high Karlovitz numbers. Combust. Theor. Model. 22, 1033-1048 (2018c)

Klein, M., Alwazzan, D., Chakraborty, N.: A direct numerical simulation analysis of pressure variation in turbulent premixed Bunsen burner flames-part 2: surface density function transport statistics. Comput. Fluids 173, 178-188 (2018d)

Kolla, H., Rogerson, J., Chakraborty, N., Swaminathan, N.: Prediction of turbulent flame speed using scalar dissipation rate. Combust. Sci. Technol. 181(3), 518-535 (2009)

Lai, J., Chakraborty, N.: Modeling of progress variable variance transport in head-on quenching of turbulent premixed flames: a direct numerical simulation analysis. Combust. Sci. Technol. 188, 1925-1950 (2016)

Langella, I., Swaminathan, N.: Unstrained and strained flamelets for LES of premixed combustion. Combust. Theor. Model. 20, 410-440 (2016)

Langella, I., Swaminathan, N., Williams, F.A., Furukawa, J.: Large-Eddy simulation of premixed combustion in the corrugated-flamelet regime. Combust. Sci. Technol. 188, 1565-1591 (2016)

Langella, I., Chen, Z.X., Swaminathan, N., Sadasivuni, S.K.: Large-eddy simulation of reacting flows in industrial gas turbine combustor. J. Propul. Power 34, 1269-1284 (2018)

Lapointe, S., Blanquart, G.: A priori filtered chemical source term modeling for LES of high Karlovitz number premixed flames. Combust. Flame 176, 500-510 (2017)

Lignell, D.O., Hewson, J.C., Chen, J.H.: A-priori analysis of conditional moment closure modeling of a temporal ethylene jet flame with soot formation using direct numerical simulation. Proc. Combust. Inst. 32, 1491-1498 (2009)

Ma, T., Stein, O., Chakraborty, N., Kempf, A.: A posteriori testing of algebraic flame surface density models for LES. Combust. Theor. Model. 17, 431-482 (2013)

Ma, T., Gao, Y., Kempf, A.M., Chakraborty, N.: Validation and implementation of algebraic LES modelling of scalar dissipation rate for reaction rate closure in turbulent premixed combustion. Combust. Flame 161, 3134-3153 (2014a)

Ma, T., Stein, O., Chakraborty, N., Kempf, A.M.: A-posteriori testing of the flame surface density transport equation for LES. Combust. Theor. Model. 18, 32-64 (2014b)

Massey, J.C., Langella, I., Swaminathan, N.: Large Eddy Simulation of a bluff body stabilised premixed flame using flamelets, Flow. Turb. Combust. 101, 973-992 (2018)

Massey, J.C., Chen, Z.X., Swaminathan, N.: Lean flame root dynamics in a gas turbine model combustor. Combust. Sci. Technol. 191, 1019-1042 (2019)

Nilsson, T., Langella, I., Doan, N.A.K., Swaminathan, N., Yu, R., Bai, X.S.: A priori analysis of sub-grid variance of a reactive scalar using DNS data of high Ka flames. Combust. Theor. Model. (2019). https ://doi.org/10.1080/13647830.2019.1600033

Peters, N.: Turbulent Combustion. Cambridge Monograph on Mechanics. Cambridge University Press, Cambridge (2000)

Pierce, C.D., Moin, P.: A dynamic model for subgrid-scale variance and dissipation rate of a conserved scalar. Phys. Fluids 10, 3041-3044 (1998)

Poinsot, T., Lele, S.K.: Boundary conditions for direct simulations of compressible viscous flows. J. Comput. Phys. 101, 104-129 (1992)

Ranjan, R., Muralidharan, B., Nagaoka, Y., Menon, S.: Subgrid-scale modeling of reaction-diffusion and scalar transport in turbulent premixed flames. Combust. Sci. Technol. 188, 1496-1537 (2016)

Reddy, H., Abraham, J.: Two-dimensional direct numerical simulation evaluation of the flame-surface density model for flames developing from an ignition kernel in lean methane/air mixtures under engine conditions. Phys. Fluids 24, 105108 (2012) 
Savard, B., Lapointe, S., Teodorczyk, A.: Numerical investigation of the effect of pressure on heat release rate in iso-octane premixed turbulent flames under conditions relevant to SI engines. Proc. Combust. Inst. 36, 3543-3549 (2017)

Selle, L.C., Bellan, J.: Scalar-dissipation modeling for passive and active scalars: a priori study using direct numerical simulation. Proc. Combust. Inst. 31, 1665-1673 (2007)

Turns, S.R.: An Introduction to Combustion: Concepts and Applications, 3rd edn. McGraw Hill, New York (2011)

Vervisch, L., Lodato, G., Domingo, P.: Reliability of Large-Eddy Simulation of nonpremixed turbulent flames: scalar dissipation rate modeling and 3D-boundary conditions. In: Meyers, J., Geurts, B.J., Sagaut, P. (eds.) Quality and Reliability of Large-Eddy Simulations Ercoftac Series, vol. 12. Springer, Dordrecht (2008)

Veynante, D., Trouvé, A., Bray, K.N.C., Mantel, T.: Gradient and counter-gradient scalar transport in turbulent premixed flames. J. Fluid Mech. 332, 263-293 (1997) 